\section{CONSIDERATIONS AS TO THE ETIOLOGY AND SIGNIFICANCE OF DILATED HEART. ${ }^{1}$}

BY H. A. CALEY, M.D., M.R.C.P. LOND.,

EHYSICIAN IN CHARGE OF OUT-PATIENTS AND LECTURER IN MATERIA MEDICA AND THERAPEUTICS, ST. MARY'S HOSPITAI.

THE object of this paper is not so much to adduce fresh facts respecting the causation of dilatation of the heart as to review the more important factors concerned in its production with especial reference to their relation to one another, to illustrate the essentially symptomatic nature of the condition, and to demonstrate that its significance in celation to prognosis and treatment depends at least as much upon its precise causation as upon its degree. Before considering cardiac dilatation in its ordinary pathological aense it is necessary to recall what modern observations have made clear as to dilatation of the heart under physiological conditions of the circulation. In the case of the healthy heart temporary dilatation may very readily be induced by increased work and within certain narrow 'limits may, as pointed out by Roy and Adami, ${ }^{2}$ Starling, ${ }^{3}$ . and others, be a mechanical advantage in relation to the ventricular output. These limits are, however, very readily passed and when this is the case the dilatation is an evidence of fatigue-it means that the heart is becoming overdone. And as in the case of other muscles so it is with the heartfatigue will manifest itself early or late according to the severity of the strain which has to be met and the condition of the muscle at the time. If the muscle is well developed and in a vigorous condition it is capable of responding within considerable limits to the call for increased work, and conversely if the muscle is from any cause enfeebled or out of condition a comparatively slight additional strain will induce rfatigue. It is broadly true of the heart as of the voluntary muscles that the liability to fatigue is dependent not on the severity and duration of the strain alone, or on the condition of the muscle alone, but on the inter-relation at the time being between the mechanical strain and the ability of the muscle to cope with the strain. In other words, in the causation of dilatation of the healthy heart which results from fatigue the two essential factorsfactors of varying relative importance-are (1) the element of mechanical strain and (2) the condition of the myocardium.

To any who have not given consideration to the subject it may not be obvious how important a bearing the temporary dilatation of the healthy heart under the stress of increased work has upon pathological dilatation. It is, however, easy to trace a gradual transition from the fugitive temporary dilatation of the vigorous heart, the result of some violent exertion-e.g., running or bicycling-to acute dilatation which is unquestionably pathological. If the strain has not been excessive and the heart muscle is healthy the disturbance of equilibrinm is but of brief, it may be almost momentary, duration, whereas if the strain has been excessive the fatigue is more lasting and it remains dilated for hours or days or weeks, as the case may be. Again, if the heart muscle is out of condition, whether from lack of training, as a result of disease, or from some other debilitating condition, a comparatively slight increase of work will cause a disturbance of equilibrium - the heart is sooner fatigued than it should be and the liability to definite pathological dilatation is great. In the former case it is the excessive work that is mainly responsible for the overstrain of the heart; in the latter it is the inability of the heart muscle to withstand the increased strain that mainly determines it. It is, however, when the two factors are combined that what under more normal conditions of the circulation would be the temporary dilatation of fatigue passes straightway into definite and more or less Jasting pathological dilatation. The importance of both factors, which we may for brevity term the mechanical and the myocardial, is unconsciously recognised in "training," which is, as is well known, mainly a matter of the heart.

1 A paper read before the Harveian Society of London on May 18th, 1899.

${ }^{2}$ Contributions to the Physiology and Pathology of the Mammalian Heart, Philosophical Transactions of the Royal Society, 1892.

3 Some Points in the Pathology of Heart Disease, THK LANCET, Feb. 27th and March 6th and 13th, 1897.
By judicious exercise the heart is gradually strengthened so that the force of its contraction is capable of effectively counterbalancing for a longer period the increase in work which is thrown upôn it during exertion, and if the increased work is sufficiently severe and long-sustained some degree of physiological hypertrophy of the heart ensues. In passing it must, however, be remarked, as emphasised by Roy, that this myocardial hypertrophy, although as strictly physiological as the hypertrophy of the muscles of the atblete or as the "blacksmith's arm" is more liable to undergo regressive metamorphosis than are unhypertrophied muscles.

But training, whilst it pays such attention to thus gradually accustoming the heart to respond to increased work, recognises the myocardial factor in other ways. In strict training just those agents and influences are eliminated which either directly or indirectly depress the heart and interfere with its muscular vigour-as, for example, alcohol and tobacco. They are avoided when the heart is to be driven at high pressure lest their tendency to cause myocardial weakness and to favour dilatation should come into play. My object in referring to these matters respecting the behaviour of the heart under physiological conditions, or under conditions which deviate comparatively little from physiological limits, is to illustrate the essential importance of my first point, that the ability of the heart to withstand the effects of extra work-or what amounts to very much the same thing, the liability of the heart to suffer from the effects of increased strain - is dependent upon the inter-relation that exists at the time being between the element of mechanical strain acting by increasing the intra-ventricular pressure and the condition of the myocardium which has in large measure to bear the strain, and that whilst on the one hand excessive mechanical strain without any important variation in the myocardial factor, and on the other primary myocardial weakness without any increase of work above the physiological mean, may, acting alone, determine a disturbance of equilibrium or definite cardiac breakdown, it is when both factors are combined that the heart is especially liable to pass from a condition of temporary heart fatigue to one of definite pathological dilatation. From which there follows as a necessary corollary the conclusion that, given a disturbance of one factor, a very slight modification of the other may suffice to turn the scale against the heart, a conclusion which underlies the fact familiar to clinicians that when the heart and circulation are in a condition of unstable equilibrium marked variations in the size and behaviour of the heart are very readily induced by comparatively trivial causes.

Reverting to the clinical etiology of pathologically dilated heart we find that when we go behind the numerous clinical antecedents of the condition the same principle still holds good; the two essential factors in its causation are: (1) increased mechanical strain, or more accurately increased intraventricular pressure ; and (2) myocardial weakness, whether primary or secondary-causes of such secondary myocardial weakness being nutritional or nervous changes, or alteration in the normal relation of the heart muscle to the pericardium. These two essential factors may be operative alone or combined; it is, however, clear that it is the rule if indeed it is not invariable, for the element of myocardial weakness to play a part sooner or later even in cases in which disturbance of the usual mechanical conditions of the circulation was the primary cause of the heart lesion.

\section{INCREASEd Mechanical Strain as a FaCtoritiN} Dilatation.

It is evident that increased intra-ventricular pressure must throw more work upon a hollow muscular organ like the heart, and the three most important proximate causes of intra-ventricular pressure so far as the left side of the heart is concerned are severe or protracted muscular exertion, abnormally high blood-pressure in the systemic circuit, and certain valvular lesions, obstructive or regurgitant, but notably aortic lesions; and for the right side of the heart sustained high blood-pressure in the pulmonic circuit, the consequence of severe mitral lesions or of pulmonary disease associated with circulatory obstruction. That such affections as these are potential causes of dilatation is an elementary fact in cardiac pathology, but we know that in fairly vigorous subjects such lesions, if chronic, are in the first instance usually productive of hypertrophy, dilatation being postponed to a later date. It is true that when hypertrophy does occur there is a certain amount, of preliminary dilatation, 
but this, except in the case of aortic regurgitation, is relatively unimportant. If, however, the strain to which the heart is subjected should be excessive or the heart muscle be unable to respond to the physiological stimulus of increased work it is primary dilatation-dilatation in the definite pathological sense - which ensues. Now it is when there is a definite tendency to dilatation that other physical factors connected with intra-ventricular pressure come into play and materially aggravate the effects of the primary mechanical difficulties with which the heart muscle has to contend. I refer to two, the importance of which was so ably demonstrated by the late Professor Roy in conjunction with Professor Adami, though hardly done justice to by clinicians. 1. That the ventricles do not normally become completely empty at the end of systole but that some residual blood remains behind, its amount being readily affected in either direction by variations in the aortic pressure. A moderate increase in the amount of residual blood does not interfere with the output of a vigorous heart but it is otherwise when the heart is already overtaxed; then the amount tends to increase and per se adds to the difficulties of the heart. Now this point is surely one of much practical importance; it means that a relatively slight increase of aortic pressure, especially so if accompanied by weakness of the systole, may by increasing the residual quantity of blood directly embarrass the heart and, though the initial departure from the normal be slight, in this way produce a cumulative effect. The recognition of this tendency will explain how in certain unstable conditions of the circulation a very moderate increase of blood-pressure or slight loss of tonus in the heart muscle may be the actual starting-point of a progressive dilatation or may greatly intensify a tendency to dilatation from some other cause. It also emphasises the potential importance of a very slight degree of pathological dilatation of the heart and confirms what clinical experience teaches-that the part played by separate etiological factors must be carefully sifted out and their influence as far as possible be eliminated if further mischief is to be prevented. 2. The second fact connected with the pliysics of the heart to which I refer is that the strain upon the walls of a sphere or spheroid increases with its circumference and therefore the resistance to contraction of the beart muscle is increased whenever it becomes dilated. This consideration, taken in conjunction with what has been said respecting the relation of increase in the residual blood, leads to the conclusion that a dilated ventricle is per se unfavourably placed for meeting increased work. It is evident, therefore, that in dealing with the element of mechanical strain in the causation of dilatation and in gauging at the bedside its importance in any given case we have to do with other factors besides the primary increase of intra-ventricular pressure. We have, so to speak, added to the primary strain certain secondary effects referable to disturbance in the physical condition of the circulation which the primary lesion has induced.

There are other factors which must not be neglected in connexion with cardiac overstrain from mechanical causesthe effect of variations in the volume of the circulating fluid, the part played by the nervous mechanism of the heart in adapting its action to varying physical conditions, and alterations of pressure in the large systemic veins, but these cannot be considered here.

Before passing on to the consideration of the myocardial factor in the causation of cardiac dilatation it may be well to refer to the fact that even in cases where the element of mechanical strain has been the primary factor the condition of the myocardium has very much to do with later developments. Thus the dilated heart of chronic high arterial tension is partly due to the rise of intra-ventricular pressure and partly to changes in the myocardium, degenerative, toxic, or the consequence of defective nutrition. In the latter connexion the precise condition of the coronary circulation is of much importance-an importance which appears to be considerably under-estimated to judge by current descriptions of heart cases. Physiologically it is well known that one of the important conditions influencing the vigour of the heart beat is the blood-flow in the coronary arteries; a rise of blood-pressure at the root of the aorta with a corresponding improvement in the coronary circulation causes increased ventricular contraction, and conversely a fall of blood-pressure in the aorta leading to a lessened blood-flow in the coronary arteries causes diminution in the force of the heart beat. So important is this relation of the coronary to the genera circulation that a general rise of blood-pressure in the systemic circuit may by increased vigour of the heart more than counterbalance the effects of the increased strain on the left ventricle which the rise of systemic blood-pressure causes, and conversely a fall of general blood-pressure by interfering with the coronary circulation may more than neutralise the effects of lessened work and lead up to myocardial failure.

It is not uncommon for us to observe at the bedside that in certain pathological states-notably chronic Bright's disease-the condition is comparatively satisfactory while the general blond-pressure remains high, whereas coincidently with the fall of blood-pressure below a certain point the heart goes to pieces. This heart failure is commonly explained as being due to the heart muscle giving out in consequence of the prolonged strain to which it has been subjected, and no doubt this is true but it appears to me extremely probable that once this diminution of general blood-pressure does supervene from commencing heart failure the progress of the latter may be greatly accelerated by a consequent fall of blood-pressure in the coronary arteries leading to imperfect nutrition of the heart muscle. Then, again, is it not probable that the marked liability to sudden heart failure in cases of advanced aortic regurgitation is in part due to the abnormally low blood-pressure in the aorta and consequent interference with the coronary circulation? Other instances might be given but enough has been said to illustrate the point that in considering the relation of variations in the general blood. pressure to the condition of the heart the relationship between the general blood-pressure in the systemic circuit and the local blood-pressure in the coronary area must be taken into account.

\section{MYOOCARDIAL WEAKNESS AS a FACTOR IN DILATATION.}

Inasmuch as in every case of dilatation of sufficient degree there is some measure of myocardial weakness which is the direct consequence of the dilatation it is necessary to remark that we are here dealing with the myocardial weakness as. the primary and predominant factor in the actual causation of the dilatation. Examples of this are the dilatation of acute specific diseases with or without actual myocarditis, fatty and other forms of degeneration, fibrosis, senile myocardial weakness, the dilated heart of severe anæmia, of nervous or general debility, and that associated with toxic causes. Clinically careful examination and inquiry usually enable us to decide as to the cases which belong to the category of primary myocardial weakness for they are generally characterised by an absence of any adequate explanation in the direction of mechanical strain, by the direct physical evidences of myocardial weakness as distinct from the associated dilatation, and by collateral evidence pointing to the operation of one of the above causes. The occurrence of more or less dilatation in acute specific diseases is well known, but there are important differences as to the degree of dilatation and the relative importance of dilatation and essential myocardial weakness. Thus in diphtheria, typhoid fever, and pneumonia, in all of which heart failure is one great source of danger, the amount of dilatation may not be great although the degree of myocardial feebleness due to the toxamia is very formidable. In this connexion the recent observations of my colleague, Dr. Lees, ${ }^{4}$ respecting dilatation of the heart in acute rheumatism are of much interest. $\mathrm{He}$ has shown, and his observations have been supplemented by those of Dr. Poynton, that the heart may be much dilated in the entire absence of pericarditis and endocarditis, that in the presence of these complications the dilatation is frequently associated rather than consequential, and that, as a rule, this dilatation is not accompanied by symptoms of actual heart failure although both during the acute phase of the illness and in more remote ways it may be of serious consequence. As an instance of how that sometimes the dilatation is conspicuous and at others myocardial weakness without much enlargement influenza may be mentioned, which is so frequently accompanied by more or less heart failure. Then, again, in the more chronic forms of dilated heart we find that the degree of dilatation is very variable and has no constant relation to the severity of the case. Fatty and granular degeneration of the heart muscle and the extreme cardiac asthenia of Addison's disease may be

4 Transactions of the Royal Medical and Chirurgical Society 
accompanied by very little variation in the size of the heart, whereas a heart with senile myocardial weakness may remain much enlarged for years with but very little in the way of serere symptoms. So important is this lack of correspondence between the amount of actual enlargement of the heart and the degree of muscular feebleness or degeneration that clinically our prognosis has to be based not so much upon the degree of dilatation as upon its cause and the amount of contractile vigour possessed by the heart muscle as eridenced by the pulse, the characters of the cardiac impulse, and of the heart sounds.

I hàve for some time past been very greatly impressed with the frequency and relative importance of toxic factors in causing or aggravating cardiac dilatation. The most striking instance of this is of course alcohol, which has so much to do with the etiology of the large number of dilated hearts seen in hospital out-patient practice. Alcohol, given in sufficient quantity under experimental conditions, acts as a direct depressant to the cardiac muscle. It causes weakening of the ventricular contractions, and if given in large enough dose induces dilatation, which may be accompanied by relative incompetence of the auriculo-ventricular valves. If we recall in this connexion that given slight dilatation any degree of superadded myocardial weakness will seriously nandicap the heart we have no difficulty in understanding the disastrous effects of alcohol on the heart and circulation. But with ordinary drinkers there are other factors besides the direct toxic action of the alcohol on the heart muscle; the large amount of fluids ingested is in itself sufficient to increase the intra-ventricular pressure, and this tendency may be intensified by the high arterial tension so often present in alcoholics. From extended and critical observation it appears to me that it is difficult to over-estimate the importance of alcoholic excess in causing or precipitating cardiac breakdown more especially in the degenerative period of life, though in speaking thus of the abuse of alcohol I do not of course under-estimate its value when judiciously employed. I said above " cause or precipitate" cardiac breakdown because alcoholic indiscretion is especially important when the heart is already overtaxed by increased mechanical strain, and when this is the case an amount of alcohol which under normal circulatory conditions would not injure the heart is sufficient to cause irreparable damage. There are many instances of this in every-day practice, such as chronic high tension and valvular lesions affecting the left side of the heart and emphysema affecting the right ventricle, in all of which failure of compensation is very liable to be prematurely induced by indiscretion in this respect.

This reference to alcohol necessitates brief allusion to other toxic agents, such as nicotine and, to a less extent, caffeine and possibly other constituents of tea. The abuse of tobacco, as is well known, causes various functional disturbances of the heart, such as cardiac pain, palpitation, tachycardia, irregularity, and the latter may if severe react unfavourably on the mechanics of the circulation; but what is of most importance in connexion with dilated heart is the direct toxic action of nicotine on the heart muscle similar to that produced by alcohol, if present in sufficient amount. I do not think that there is much clinical evidence that tobacco is a relatively important factor by itself in causing dilatation, but I am convinced from clinical observation that when the heart is already embarrassed indiscretion in this respect is highly injurious, producing just that lack of tone in the heart muscle which in the presence of mechanical embarrassment is enough to disturb compensation. Having alluded to the influence on the heart of the poisons of infective diseases and of certain known chemical poisons introduced from without, such as alcohol and nicotine, I must mention that it seems highly probable that toxæmic agents of another kind may also under certain circumstances directly depress the heart as well as indirectly through causing high tension. I refer to auto-intoxication of gastro-intestinal origin. At present the relation of this complex subject to the condition of the circulation is one for observation rather than deduction, but the clinical fact that a marked improvement in the strength of the heart's action at times coincides with the cessation of abnormal fermentative changes in the digestive tract is not without its significance and is not by any means always explicable as being due to relief of pressure. This consideration of the part played by toxic agents in causing or aggravating a tendency to dilatation and the wider question of the relation of the condition of the heart muscle in general to dilatation, alone or in conjunction with the element of increased mechanical strain. leads up to a fresh enunciation on pathological grounds of the principle to which prominence has already been given mainly on physiological grounds, that the essential factor in the etiology of dilatation is the relation that subsists in any given case between the work which the heart has to do and the condition-structural and functional-of the myocardium at the time being, so that in disease while alterations in the mechanical conditions of the circulation alone or primary weakness of the heart muscle alone may induce dilatation, in a very large proportion of cases when serious dilatation ensues both factors are combined, though in very variable relative proportions and with marked differences as to dates of incidence.

No more striking instance of this inter-relation could be given than that afforded by heart diseases in which alterations in the physical conditions of the circulation play an obviously important part. Take two examples-mitral regurgitation associated with rheumatic endocarditis and chronic high arterial tension. In this form of mitral regurgitation it is, as is well known, the rule, if there is good compensation, to find the left ventricle dilated and hypertrophied and the right ventricle also; and a certain amount of äilatation, always provided it is accompanied by sufficient hypertrophy, is an advantage in that it conduces to increased output on the part of the left ventricle and to the projection into the aorta of a sufficient volume of blood, notwithstanding the leakage through the mitral orifice. In some cases of mitral disease, however, the amount of mitral regurgitation is out of proportion to the damage to the valve structures found post mortem; when that is so there is usually marked dilatation of the left ventricle and we are justified in concluding that the excess of mitral regurgitation over and above that due to the sclerotic changes in the valves set up by the endocarditis is referable to added incompetence from relaxation of the circular band of muscular fibres, which forms a kind of sphincter for the auriculo-ventricular orifice, so that even in a case of mitral regurgitation associated with structural deformity of the valve from endocarditis myocardial weakness with associated dilatation plays a highly important part in the cardiac embarrassment. If severe this adds to the circulatory embarrassment in another way, it leads to a fall of bloodpressure in the systemic arteries, for the much-dilated and weakened left ventricle cannot satisfactorily maintain an adequate output with each heart beat. Precisely the same point is illustrated by the effects of treatment in cases of severe mitral regurgitation with failing compensation. With absolute rest, measures to relieve the venous engorgement and digitalis, compensation is restored. The symptoms and effects of back-working diminish, the heart becomes more regular, the cardiac dulness shrinks, the impulse becomes more forcible, and the amount of mitral regurgitation lessens-the diseased valve remains unaltered, but the heart muscle has regained its vigour.

So important is this muscular action in relation to the auriculo-ventricular orifice that, as Starling points out, it is probable that so long as the circular band of muscular fibres surrounding the orifice is intact perforation of the valve does not cause much disturbance. It is when fatigue of the heart muscle sets in or the orifice is held firmly by sclerosed connective tissue that severe incompetence occurs. The second example of the importance of the myocardial factor in conditions in which the element of increased mechanical strain plays the chief part in etiology was chronic arterial tension. Of this we need only remark that the conspicuous variations in different cases in the behaviour of the heart under the stress of increased strain of peripheral origin are intimately dependent upon differences in the ability of the myocardium to adapt itself to the altered blood-pressure and thus maintain compensation for a longer or shorter time and that it is frequently possible to trace the onset of failure of compensation to the supervention of definite myocardial weakness due to toxic or nutritional causes.

\section{The Signiftcance of Dilated Heart.}

Leaving aside strictly physiological distension of the cavities of the heart and strictly compensatory dilatation as in some valvular lesions the general significance of dilatation is that there is or has been a disturbance in the relation which should subsist between the work which the heart has to do and the power of the heart muscle to perform it. Physiological dilatation is (beyond certain narrow limits) an evidence of heart fatigue, pathological dilatation is an evidence of heart over-strain, and frequently the precursor 
of heart failure. But what concerns us from the point of view of prognosis and treatment is the special significance in an individual case of any cardiac dilatation that may be present. This we can best ascertain by attention to the following points.

1. The degree of dilatation.-Now, as Roy and Adami pointed out, under physiological conditions dilatation is the equivalent of fatigue, so that the measure of the dilatation is a reliable indication of the degree of fatigue. From this the deduction is drawn that in "a diseased heart the degree of dilatation is in inverse ratio to its power of doing its work." In diseases of the heart we certainly do find that, other things being equal, the more severe the dilatation the greater the embarrassment of the heart, and it is no doubt broadly true that the degree of dilatation is in inverse ratio to its power of doing its work, but the converse of this certainly does not hold good owing to the fact that under pathological conditions the other things are not equal; and the further we depart from the comparatively simple conditions of a normal heart yielding under the stress of increased work the less reliance can we place on the degree of dilatation as in itself a gauge of the significance of the lesion. We must take note of other factors and the next one to be considered is-

2. The condition of the myocardium with which the dilatation is associated. - It is well known that a man with fatty degeneration or syphilitic fibrosis or gumma of the heart walls, or with disease of the coronary arteries, may be on the brink of a catastrophe although the heart is but little dilated, whilst another with much enlargement of the heart, including considerable dilatation, may have years of life and usefulness before him. In the one case the heart may suddenly give out without any definite exciting cause; in the other so long as the work thrown upon it is kept within bounds it will continue to perform its functions for a prolonged period. The determining factor is the myocardial one, the structural and functional condition of the myocardium. And in so far as we can estimate the amount of contractile vigour which the heart muscle possesses we must regard it as important, in itself and also as an evidence of neuro-muscular tone. Having ascertained the degree of dilatation and the condition of the heart muscle with which it is associated we pass on to the third factor

3. The precise etiology of the dilatation, with especial reference to the relation of the heart condition to the general health, the relative importance of the mechanical and myocardial factors in its production, and whether one or both of these factors is still operative.-In some cases both factors have been largely responsible and their precise relationship is not of very great importance-e.g., the severe dilatation of anæmia in which the muscular feebleness incidental to the blood state has been combined with injudicious exertion, but in others when one of the two factors has been in the ascendant it is highly important to recognise this. For instance, the significance of the acutely dilated heart from athletic overstrain in a young man is very different from the acute dilatation induced by violent exertion in a man of advanced years or one whose health has been impaired by alcoholic excess. Or, again, we regard very differently moderate dilatation associated with chronic high tension and the same degree of dilatation due to senile myocardial feebleness. The further question as to whether one or both factors has ceased to be operative is closely connected with this. The dilated heart of athletic over-strain when adequate rest is assured we may expect to do well because the causal condition is no longer acting and the heart is capable of pulling itself together, but we cannot say the same of a similar degree of dilatation associated with the persistent strain of chronic high tension in renal disease. Likewise, we may be able confidently to predict restoration of tone to the heart muscle which has been temporarily depressed by some toxic agency no longer operative, whereas it is far otherwise with the myocardial weakness of senile or degenerative origin. Our next factor in prognosis is derived not from a consideration of the anatomical conditions of the heart but of the extent to which, abnormal as it is, it is capable of carrying on its primary physiological functions in relation to the general circulation. We accordingly inquire as to-

4. The extent to which the work of the heart in relation to general circulation is affected.-Apart from physical signs this we chiefly estimate by noting any definite cardiac symptoms or any manifestations of backward pressure on the v.nous side. This is the basis of what we must all recognise, that in dealing with heart cases it is necessary to weigb symptoms and physical signs together, and that not uncommonly the former teach us more than the latter as to the extent to which the cardiac lesion is interfering with its work. The last point to which I would direct attention is-

5. The course of the case and the effect of treatment.-Just as the test of the physiological integrity of a heart temporarily dilated by excessive strain is the readiness with which it resumes its normal size and action when the stress has been removed, so the test of recuperative power on the part of the pathologically dilated heart is its ability to react to therapeutic measures. Dilatation which rapidly diminishes under strict rest or which improves with graduated exercise is evidently of less serious import than one which requires prolonged rest and powerful cardiac tonics. The converse of this is nowhere better illustrated than in chronic valvular disease. With each successive breakdown of compensation there is less and less response to therapeutic measures until a stage is reached (no doubt coincident with irreparable myocardial failure) when precisely the same measures fail to make any impression - a sure sign that dissolution is not far distant.

The matters to which I have given prominence may be thus summarised: that in the etiology of cardiac dilatation the two essential factors, factors of varying relative importance, are (1) the element of increased mechanical strain, and (2) myocardial weakness, primary or secondary; that the liability of the heart to suffer from the effects of increased work is dependent upon the inter-relation that exists at the time being between the element of mechanical strain and the condition of the myocardium which has in large measure to stand the strain, and that it is when increased mechanical strain and myocardial weakness are combined that there is the greatest liability to severe cardiac dilatation. We have seen that a dilated ventricle is per se unfavourably placed for coping with increased work, that given a slight degree of dilatation from mechanical causes toxic and other agencies which depress the heart muscle may greatly intensify the cardiac embarrassment, and that in a large proportion of cases in which alterations in the mechanical conditions of the circulation constitute the primary factor in the production of dilatation its later progressive development is attributable to the supervention of myocardial changes. Then with respect to the significance of the dilatation that just as physiological dilatation (beyond certain narrow limits) is an evidence of heart fatigue, pathological dilatation is an evidence of heart overstrain-past or present-and that in order to estimate its significance with any degree of accuracy we must take note of the degree of dilatation, the condition of the heart muscle with which the dilatation is associated, its precise etiology, the extent to which the work of the beart in relation to the general circulation is affected by it, and the amount of recuperative power which the heart, and we may add the individual, possesses, this recuperative power being evidenced by the course of the case and its reaction to treatment.

A broad view on lines such as these is essential if we are to avoid the extremes of making too much or too little of any given cardiac dilatation, and perhaps at the present time the former is as important as the latter.

Upper Berkeley-street, $\mathbf{W}$.

Medical Town Counctulor.-On May 25th, at a municipal election at Bath, Dr. G. A. Bannatyne was returned by a large majority as representative of the Landsdown Ward.

Presentations to Medical Men, - Mr. James McKay, M.B., Ch.B. Aberd., of Towie, has been presented by the members of the local class under the St. Andrews Ambulance Association with a silver kettle with a spirit-lamp as an acknowiedgment of his first-aid instructions to the class. - At Blackburn, the ambulance class conducted by Mr. Edward Oliphant, M.B., C.M. Aberd., was brought to a close by an "At home," held in the Drill-hall, on May 19th, when the certificates were handed to the successful members, following which Dr. Oliphant was the recipient from the class of a marble timepiece in recognition of his services.-Mr. P. E. Corlis, M.D., Wooster, Cleveland, U.S.A., was entertained at a conversazione by the citizens of Ballina, New South Wales, and presented by them with two addresses prior to his leaving that city. 


\section{ANEURYSM OF THE SUBCLAVIAN ARTERY; LIGATURE OF THE FIRST POR'IION OF THE SUBCLAVIAN, FOLLOWED IN THIRTY-EIGHT DAYS BY REMOVAL OF THE ANEURYSM.}

BX HERBERT W. ALLINGHAM, F.R.C.S. ENG., SURGEON TO THE HOUSEHOLD OF H.R.H. THE PRTNCE OF WALES ; ASSISTANT SURGEON AND LECTURER ON OPERATIVR SURGERY AT ST. GEORGE'S HOSPITAI.

A SEAMAN invalided from the Royal Navy, aged 25 years, was admitted into St. George's Hospital on Feb. 20th, 1899, and gave the following history. Three years previously he had noticed a swelling above the right clavicle which increased gradually in size. This increase had been especially marked during the 12 months previously to the date of his admission and during this period he had suffered from occasional attacks of numbness and severe aching pain in the right upper extremity. The pain, which at first occurred only at intervals, had lately become continuous without being accompanied at any time by swelling below the tumour. The patient's general health had always been good and syphilis was denied. On examination the patient was obviously in good health and of good muscular development. He exhibited a pulsating expansile swelling in the right supra-clavicular region, filling the interval between the sterno-mastoid and trapezius muscles, and in size of about that of a Tangerine orange. There was a characteristic systolic bruit with delay and diminution of the right radial pulse. Some slight tenderness on pressure over the tumour was evident, but otherwise there was no symptom except the pain passing down the inner side of the arm and forearm to the little finger. A consultation with my colleagues was held and it was decided to ligature the subclavian artery on the cardiac side of the tumour. This was done on March 1st through a V-shaped incision, one arm of the $\mathrm{V}$ being down the anterior border of the sterno-mastoid and the other arm along the clavicle. The flap of skin was turned up. The sterno-mastoid muscle was divided and the first part of the subclavian artery was exposed and a kangaroo tendon passed round the vessel immediately external to the origin of the vertebral artery and just to the inner border of the scalenus anticus. The recovery from this operation was complete and at no time was there any indication of secondary hæmorrhage. No pulsation was felt in the tumour or in the brachial or radial arteries. On the 4th some slight beating was detected in the aneurysm, which continued, its amount being carefully noted day by day. The sac meanwhile was distinctly smaller and harder. On the $17 \mathrm{th}$, as the pulsation was still decided, the patient was again anæsthetised and a further consultation was held. It was found that the tumour although pulsating did not expand with the beat of the heart and it was therefore considered advisable to further watch its progress before attempting more radical measures. On the 28th it was noted that the aneurysm was increasing in an upward direction and that there was more pulsation which was both visible and palpable. On April 8th, my colleagues, Mr. Holmes, Mr. Dent, and Mr. Turner, to whom I am much indebted for their valuable advice, being present, and assisted by my colleague Mr. Jaffrey and Mr. Collard, the radical operation was performed. The whole length of the clavicle was exposed by a transverse incision and in order to get plenty of room to deal with the aneurysm a vertical incision was made at right angles to the transverse incision. Then large skin flaps were reflected and the greater part of the clavicle was resected, leaving only the two extremities. Careful dissection revealed the aneurysm springing from the upper and anterior aspect of the vessel in its third portion. With great care the cellular tissue, the nerves, and the vessels surrounding the aneurysm were dissected off the sac. Then the axillary artery was sought for, ligatured with silk, and divided on the distal side of the tumour. This procedure allowed the aneurysm to be freed from the first rib and turned upwards and so to bring into view the second part of the subclavian artery on the proximal side of the aneurysm. This part of the artery was ligatured with silk and the aneurysm was cut away. During the dissection the transversalis colli artery was divided as it crossed the tumour and some few fibres from the brachial plexus passing into the pectoralis major muscle were also divided. The lower part of the brachial plexus was adherent to the upper part of the tumour and had to be dissected off it. Some difficulty was experienced in separating the subclavian vein which received a slight tear, necessitating a lateral ligature. The amount of blood lost was quite incon. siderable, notwithstanding the number of vessels divided, owing to the establishment of so free a collateral circula. tion. The operation occupied two hours. The after-history was unimportant, healing of the large wound taking place after some slight local skin sloughing. A section of the

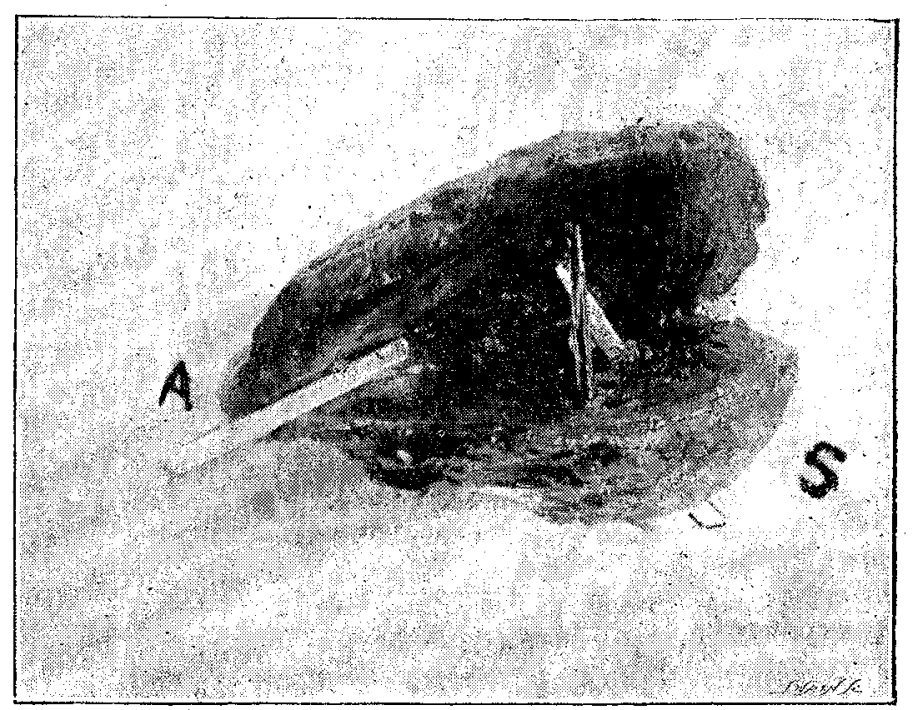

Figure sbowing sac of aneurysm removed from the subclavian artery. A, Axillary artery. s, Subclavian artery.

aneurysm shows that its cavity is largely occupied by firm clot, the blood passing into it from the subclavian, which was partly patent, and out posteriorly by way of what appeared to be the profunda cervicis. In shape it resembled a pear with the stalk external and the base upwards. The axillary artery, as far as it was exposed in the wound, was found to be obliterated. A careful examination of the arm was made on May 5 th, and it was found that pulsation was present in the brachial artery, best marked in the ante-cubital space and also in the radial at the wrist. The ulnar was not felt. The hand and fingers were somewhat pallid and colder than those of the opposite limb; this condition, however, is undergoing improvement from day to day.

Remarks.-There are many points of interest in this case, not the least of which is the fact that it is apparently unique as far as the measures adopted for its relief are concerned, a search through published records having revealed no similar case. To take them separately. First, the ligature of the first portion of the main vessel was followed by decidedly good results, limited though these were to a hardening of the aneurysm and diminution in its pulsation. It is interesting, also, that the procedure was not followed by secondary hæmorrhage as is supposed to follow on ligature of the first part of the subclavian artery. As there was no prospect that the pulsation would cease altogether, the difficult and severe expedient of removal was resorted to five and a half weeks after the preliminary operation. Here it may be remarked that the hardening and, if one may so put it, the better definition of the tumour brought about by a previous ligature of the subclavian rendered the complete removal easier and more expeditions than if a soft, thin-walled sac had had to be dealt with, in addition to the probability that had rupture accidentally occurred during manipulation loss of blood would have been less excessive owing to the small calibre of the channel through the aneurysm. Secondly, the preliminary ligature had caused the collateral anastomosing channels to become active that fear of gangrene was at no time entertained. Thirdly, removal of the greater part of the clavicle was necessary in this case, access to the sac and to the vessel below being impossible without this operation. Even now, so soon after the operation, the patient can perform all of the usual movements without difficulty, although it can hardly be expected that he will retain full 